\section{Revue de l'IFHA}

Revue de l'Institut français d'histoire en Allemagne

$1 \mid 2009$

IFHA 1

\title{
La mémoire des origines dans les ordres religieux- militaires au Moyen Âge / Die Erinnerung an die eigenen Ursprünge in den geistlichen Ritterorden im Mittelalter
}

Journée d'études internationale, Göttingen, 25-26 juin 2009

\section{Mathieu Olivier}

\section{OpenEdition}

Journals

Édition électronique

URL : http://journals.openedition.org/ifha/334

DOI : 10.4000/ifha.334

ISSN : 2198-8943

Éditeur

IFRA - Institut franco-allemand (sciences historiques et sociales)

\section{Édition imprimée}

Date de publication : 30 septembre 2009

Pagination : 103-107

ISSN : 2190-0078

Référence électronique

Mathieu Olivier, "La mémoire des origines dans les ordres religieux-militaires au Moyen Âge / Die Erinnerung an die eigenen Ursprünge in den geistlichen Ritterorden im Mittelalter », Revue de I'IFHA [En ligne], 1 | 2009, mis en ligne le 07 février 2013, consulté le 15 septembre 2020. URL : http:// journals.openedition.org/ifha/334

Ce document a été généré automatiquement le 15 septembre 2020

(CIFHA 


\section{La mémoire des origines dans les ordres religieux-militaires au Moyen Âge / Die Erinnerung an die eigenen Ursprünge in den geistlichen Ritterorden im Mittelalter}

Journée d'études internationale, Göttingen, 25-26 juin 2009

Mathieu Olivier

\section{NOTE DE L'ÉDITEUR}

Rapport établi par Mathieu Olivier

Avec le soutien financier de la MHFA, Mathieu Olivier (MHFA) et Philippe Josserand (Université de Nantes, CRHIA) ont organisé les 25 et 26 juin 2009 journées d'études sur le thème de la mémoire des origines dans les ordres religieux-militaires au Moyen Âge. La recherche des origines possède dans l'historiographie de chacun des différents ordres religieux-militaires une longue tradition érudite. Elle n'a pas été sans nourrir d'infinies controverses, dont les raisons sont à chercher dans les obstacles qui se dressent devant l'historien dans l'appréhension des premiers temps des communautés considérées. Si le premier écueil est bien, très souvent, l'existence d'une documentation lacunaire, un second a également contribué à obscurcir la vision des débuts: la persistance de mythes et légendes d'origines divers, dont il a fallu au préalable se délester. L'un des apports essentiels des travaux sur les ordres religieux-militaires depuis un demi-siècle aura précisément été de passer au crible ces discours fallacieux et de les dévoiler par là même pour ce qu'ils sont le plus souvent: des mémoires des origines sinon toujours controuvées, du moins construites après coup. Alors que le temps de la critique des (principaux) textes, pour l'essentiel, est derrière nous, c'est 
tout naturellement qu'un nouveau type de questionnement se fait jour, qui porte sur les raisons d'être et les conditions de possibilité de l'apparition de telles mémoires dans le contexte des ordres religieux-militaires jusqu'à l'aube du XVIe siècle. Il ne s'agissait donc pas de réhabiliter subrepticement des traditions frauduleuses que même une réfutation formelle eut bien du mal à réduire au silence - pour reprendre les termes d'A. Forey ${ }^{1}-$, mais bien d'interroger pour elle-même, et non pour sa valeur informative, cette mémoire des origines.

2 Les « récits d'origines » ont depuis toujours fait l'objet d'une attention privilégiée ; il serait pourtant réducteur de s'en tenir à ce support narratif. Aussi avait-t-il semblé pertinent de préférer la notion plus englobante de "mémoire des origines », notion dans laquelle la memoria liturgique aussi bien que le support iconographique - des sceaux à l'héraldique - ou encore les mécanismes de conservation archivistique des actes fondateurs, par exemple, ont toute leur place. Deux raisons motivèrent cet élargissement de l'horizon de l'enquête. Il s'agissait tout d'abord d'éviter par là même de couper d'emblée ces textes d'une culture mémorielle plus large dont ils seraient telle était l'hypothèse de travail retenue - en quelque sorte la partie émergée, et sans laquelle ces récits ne seraient guère compréhensibles. Ce choix résultait en outre du constat, motif constant d'étonnement comme de frustration, de l'extrême disproportion des sources narratives d'un ordre à l'autre, et notamment de la surreprésentation écrasante, dans cette catégorie, des matériaux en provenance de l'ordre Teutonique. S'il serait malvenu de renoncer à questionner cette disparité ellemême, seule la mobilisation simultanée de tous les vestiges de la culture mémorielle des ordres religieux-militaires est à même de contourner la difficulté et d'ouvrir à la comparaison des perspectives véritablement prometteuses.

3 Par son objet comme par ses partis pris méthodologiques, cette journée d'études ambitionnait de s'inscrire à l'intersection de deux domaines de recherches dont le dynamisme ne se dément pas. Elle se concevait d'une part comme le prolongement du regain d'intérêt pour les pratiques de l'écrit dans les ordres religieux-militaires thème général du colloque Ordines Militares tenu à Toruf en septembre 2007. Elle émergeait d'autre part du débat autour de la " conscience de soi » de ces communautés et de ses avatars au cours du bas Moyen Âge, débat qui s'est intensifié depuis une quinzaine d'années parmi les spécialistes.

4 Il n'était pas question, dans le cadre de cette journée d'études, de prétendre faire le tour de ces interrogations. Le dessein premier était plutôt de faire le point des acquis et de confronter les travaux en cours, ainsi que d'ouvrir un chantier d'inventorisation de la documentation pertinente dans l'optique que nous occupe ici. La rencontre de Göttingen espérait de la sorte poser quelques jalons pour des recherches futures, à l'heure où l'intérêt de faire dialoguer des historiographies longtemps cloisonnées à l'extrême est devenu une évidence unanimement reconnue.

5 La manifestation s'est déroulée dans le cadre de la salle de conférences de la MHFA à Göttingen et a tenu toutes les promesses que laissait entrevoir un programme riche, fruit de l'écho très positif rencontré par le thème des journées d'études auprès des douze médiévistes français, allemands, polonais et portugais invités à y prendre part. Après le mot de bienvenue prononcé par Thomas Lienhard, directeur de la MHFA, M. Olivier a rapidement rappelé, en guise d'entrée en matière, le contexte historiographique de la question, ainsi que les idées directrices qui présidèrent à la conception de ces deux journées. Il céda ensuite la parole aux premiers orateurs pour 
trois communications à visée introductive. Ouvrant la session, Florent Cygler (Université de Nantes) a brossé un tableau de l'état de la recherche sur le discours des origines dans les ordres monastiques et mendiants au Moyen Âge, revenant sur les principaux enjeux de la construction des origines dans le processus d'institutionnalisation des communautés régulières. Doublement intéressant en sa qualité d'ordre hiérosolymitain et de matrice des "proto-Templiers", le cas des chanoines du Saint-Sépulcre a ensuite été étudié par Waldemar Könighaus (Akademie der Wissenschaften, Göttingen). Dernier temps de cette session introductive, la communication de Jürgen Sarnowsky (Universität Hamburg) a très utilement permis de faire un vaste tour d'horizon des sources historiographiques (au sens le plus large du texte) produites par les ordres militaires du XIIe à l'aube du XVIe siècle.

6 L'après-midi du jeudi 25 fut consacré aux récits d'origines, forme la plus classique et la plus étudiée de l'invention de la tradition. Antoine Calvet (Université Paris IV) est revenu sur les légendes d'origines de l'ordre de Saint-Jean, complexe textuel foisonnant, plurilingue et encore mal connu dans le détail. En guise de récit médiéval d'origine de l'ordre de Saint-Lazare, on ne dispose guère que d'une brève et énigmatique mention dans une description de Jérusalem remontant au XIIe siècle : par une patiente enquête régressive à partir de narrations du début de l'époque moderne, couplée à un savant travail de mise en relation d'éléments épars dans la documentation, François-Olivier Touati (Université François-Rabelais, Tours) est parvenu à tracer les contours d'une mémoire des origines en forme de constellation de références plus que de narration historique. Mêlant faux diplômes, mythe d'origine voué à une certaine fortune historiographique, et plusieurs essais de recomposition du récit de fondation à l'extrême fin du XVe siècle, le dossier santiaguiste se distingue par sa richesse au sein des ordres ibériques: Philippe Josserand (Université de Nantes) l'a parcouru en connaisseur, s'attardant particulièrement sur une étonnante compilation de 1481, la chronique de Diego Rodriguez de Almela (Compilación de los milagros de Santiago). La Narratio de primordiis de ordinis Theutonici (XIIIe siècle) et les autres récits de fondation de l'ordre Teutonique gravitant dans l'orbite de cette relation centrale, furent au centre des deux dernières communications de la journée, celles d'Udo Arnold (Rheinische Friedrich-Wilhelms-Universität, Bonn) et de Jarosław Wenta (Uniwersytet Mikołaja Kopernika, Toruf). Il en ressort un faisceau d'hypothèses nouvelles et partiellement concurrentes, tant sur la question, difficile, de la datation du texte que des circonstances de sa genèse.

7 L'ambition de la rencontre était d'aller traquer la conscience des origines par-delà ces récits ; les quatre communications de la seconde journée s'employèrent à lui donner un contenu. Alain Demurger (Université Paris I) s'est interrogé sur une absence maintes fois relevée : pour autant que l'on puisse en juger, les Templiers n'élaborèrent aucun récit d'origine comparable aux Légendes hospitalières ou à la Narratio teutonique; on ne saurait pour autant en conclure que la conscience de soi templière ne s'ancrait pas dans une vision forte des origines de la communauté. Consacrée au dossier sur les donations des comtes de la maison d'Urgell-Forcalquier dans les archives hospitalières de Manosque et de Saint-Gilles, la communication de Damien Carraz (Université de Clermont-Ferrand II) fut l'occasion d'aborder deux dimensions encore insuffisamment explorées jusqu'à présent : celle de l'échelon local (commanderie) et de la mémoire des origines qui s'y rattache tout d'abord; celle de la «mémoire documentaire » ensuite. Sylvain Gouguenheim (ENS LSH, Lyon) a prolongé cette réflexion sur la mémoire documentaire par le biais de l'analyse d'un « diplôme fondateur » de l'ordre Teutonique 
et de sa mémoire à la fin du Moyen Âge : la bulle Etsi neque (1220). Enfin, c'est à une plongée au cœur de l'invocation pragmatique des origines dans la correspondance diplomatique de l'ordre Teutonique du XVe siècle que nous a conviés Annika Souhr (Universität Hamburg) en point d'orgue de ces journées, avant que M. Olivier (MHFA) ne propose quelques éléments de conclusion dans la foulée des dernières interventions. Au terme de près de deux jours de contributions riches et de discussions animées, le vœu de donner de multiples prolongements à ce ballon d'essai a été partagé par l'ensemble des participants. Le premier, dont les modalités exactes restent à déterminer, devrait être d'ordre éditorial, sous forme de la publication des actes, unanimement reconnue comme un aboutissement souhaitable des journées d'études.

9 Axel Ehlers (Hanovre), Thomas Krämer (Freie Universität Berlin), Thomas Lienhard (MHFA), Olivier Richard (Université de Haute-Alsace, Mulhouse) et Frédéric Vitoux (ENS LSH Lyon) ont également pris une part active à la discussion. Ivan Ivanov (Universität Göttingen/ MHFA) et Susanne Wiesenthal (MHFA) ont apporté une contribution décisive au travail préliminaire de traduction en allemand des textes fournis par les intervenants francophones.

\section{NOTES}

1. Alan Forey, "The Emergence of the Military Orders in the $12^{\text {th }}$ century " in : Journal of Ecclesiastical History, 36/2, 1985, p. 175. 